\title{
Una «hazaña prácticamente desconocida»: la participación de médicos militares españoles en la Primera Guerra Mundial
}

\author{
Navarro Suay R. ${ }^{1}$, Plaza Torres JF. ${ }^{2}$
}

Sanid. mil. 2014; 70 (1): 51-57; ISSN: 1887-8571

\begin{abstract}
RESUMEN
La Primera Guerra Mundial fue un conflicto que tuvo lugar entre 1914 y 1918. Debido a la situación interna y en el exterior de España, el rey Alfonso XIII y el presidente Eduardo Dato decidieron mantener a nuestro país neutral. Sin embargo, se consiguió realizar una conducta «humanitariamente activa». Se creó la oficina pro cautivos, se colaboró con el comité internacional de la cruz roja, se enviaron observadores militares (entre ellos oficiales médicos) al frente europeo y se autorizó la asistencia a refugiados alemanes en la Guinea Ecuatorial Española. El objetivo de este artículo es describir la labor realizada por los oficiales médicos españoles desplegados en calidad de observadores en el frente europeo durante la primera guerra mundial, destacando como ejemplo de ellos a los capitanes médicos Mariano Gómez Ulla, Fidel Pagés Miravé y Antonio Vallejo Nágera. Así mismo, se analiza la asistencia sanitaria prestada a refugiados alemanes en la por entonces colonia española de Guinea durante ese mismo periodo.
\end{abstract}

PALABRAS CLAVE: Medicina militar, primera guerra mundial, observador, Guinea.

An unknown history: The spanish military doctors participation in the first world war.

SUMMARY: First world war was a conflict that took place between 1914 and 1918. Due to internal and external situation, king Alfonso XIII and the spanish president Eduardo Dato decided keep Spain like a neutral country. However, the spanish position was active from the humanitarian point of view. It was created a pro captives office, Spain collaborated with the international red cross, some military observer were sent to the european frontline and german refugees were attended in Guinea. The aim of the study is to describe the work of the spanish medical officers deployed like observers in the european frontline during first world war (i.e Captains Mariano Gómez Ulla, Fidel Pagés Miravé and Antonio Vallejo Nágera). We have analized too, the health care provided to german refugees in Guinea (in those days Spanish colonie) during the same period.

KEY WORDS: Military medicine, First World War, Observer, Guinea.

\section{INTRODUCCIÓN}

La primera guerra mundial fue un conflicto que tuvo lugar entre 1914 y 1918. La guerra comenzó como un enfrentamiento entre el imperio Austrohúngaro y Serbia. Las hostilidades involucraron a dos coaliciones de países, los denominados «aliados»: Francia, Reino Unido, Rusia, Serbia, Bélgica, Canadá, Portugal, Japón, Estados Unidos (desde 1917) e Italia, que se enfrentaron a la coalición de las «potencias centrales», integrada por Bulgaria y los imperios austrohúngaro, alemán y otomano. Debido a las diferentes alianzas entre gobiernos, el conflicto comenzó en Europa y se extendió por las respectivas colonias de África y de Asia. Tras cuatro años de guerra, los países «aliados» salieron victoriosos firmándose finalmente la paz en el tratado de Versalles de 1919 $9^{1,2}$.

La neutralidad española durante la primera guerra mundial fue iniciativa del rey Alfonso XIII (Figura 1) y del presidente

\footnotetext{
Cte. Médico. Hospital Central de la Defensa «Gómez Ulla», Servicio de Anestesiología y Reanimación, Madrid. España.

2 Cte. Médico. Escuela de Suboficiales de la Armada, Servicio de Sanidad, San Fernando. Cádiz. España.
}

Dirección para correspondencia: Ricardo Navarro Suay. Servicio de Anestesiología y Reanimación. Hospital Central de la Defensa «Gómez Ulla». Glorieta del Ejército s/n. 28047 Madrid (España). E-mail: r navarro suay@yahoo.es

Recibido: 6 de mayo de 2012

Aceptado: 9 de septiembre de 2013 de gobierno Eduardo Dato. La debilidad militar tras el desastre de 1898 , las tensiones políticas en el interior del país, la guerra colonial en el norte de Marruecos y las buenas relaciones personales entre el rey Alfonso XIII y las diferentes monarquías de los países beligerantes originaron que nuestro país se mantuviera oficialmente neutral. Sin embargo, esta política no fue una postura de indiferencia frente a la guerra. Nuestro monarca visitó el frente, creó la oficina pro cautivos en las dependencias del palacio real, colaboró estrechamente con el comité internacional de la cruz roja, permitió la presencia de observadores militares en los campos de prisioneros de ambos bandos ${ }^{3}$ y autorizó la asistencia humanitaria de refugiados en nuestro territorio ${ }^{4}$.

El objetivo de este artículo es describir la labor realizada por los oficiales médicos españoles desplegados en calidad de observadores en el frente europeo durante la primera guerra mundial y analizar la asistencia sanitaria prestada a refugiados alemanes en la por entonces colonia española de Guinea durante ese mismo periodo.

\section{MÉDICOS MILITARES ESPAÑOLES EN EL FRENTE EUROPEO}

Muchos militares participaron en las tareas encomendadas por el Rey Alfonso XIII para la inspección de campamentos de 


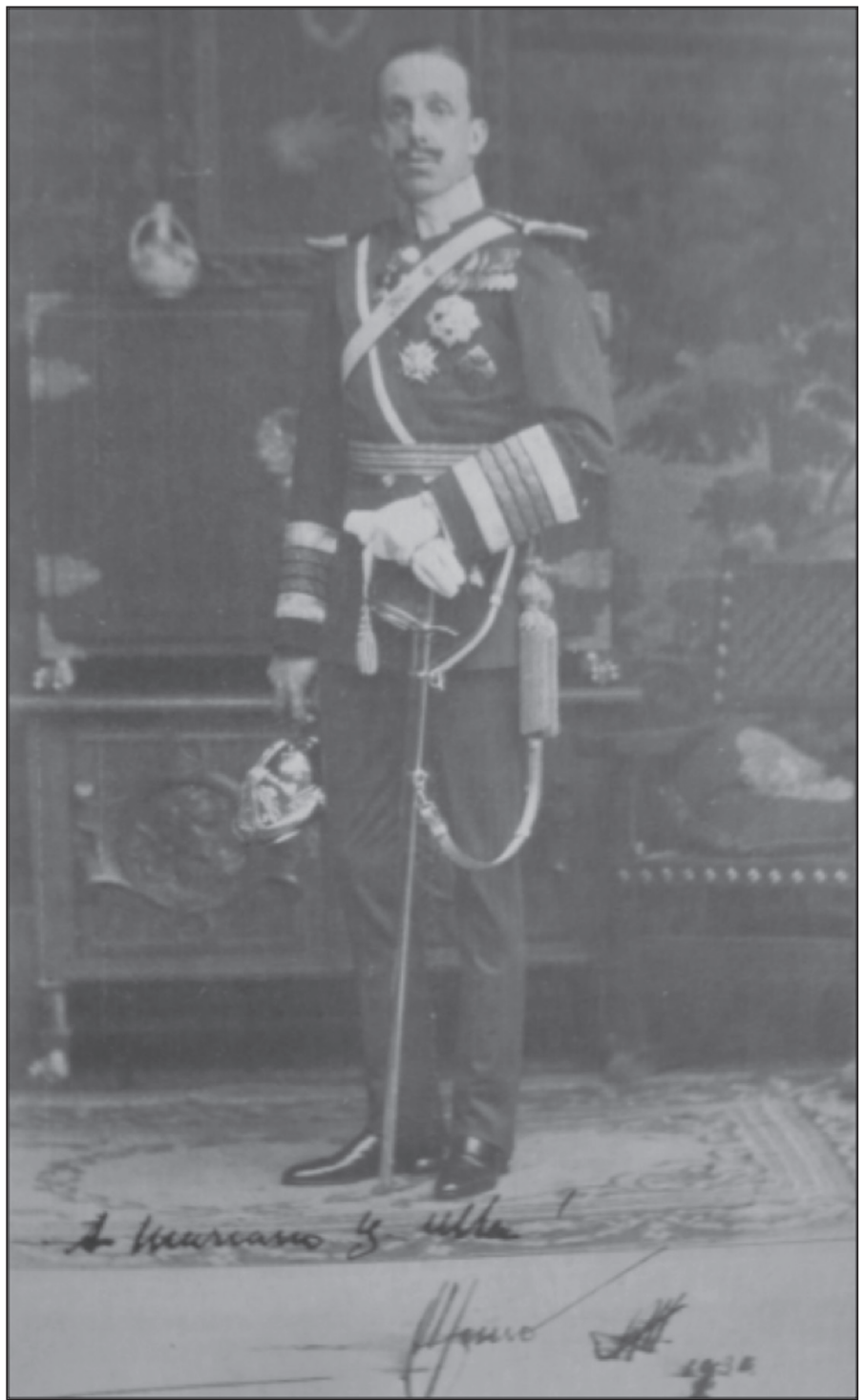

Figura 1. Fotografía de Su Majestad el Rey Alfonso XIII dedicada a Mariano Gómez Ulla. Imagen tomada de Gómez Ulla M. Mariano Gómez Ulla: un hombre, un cirujano, un militar. Madrid: 1981.

prisioneros en Europa durante el conflicto bélico. La mayoría pertenecían a las distintas Armas del Ejército y a la Armada. Sin embargo, también se comisionaron a oficiales médicos ${ }^{5}$. La condición de militar, su imprescindible formación facultativa y el conocimiento de idiomas fueron determinantes en esta decisión humanitaria.

La participación de oficiales médicos españoles se puede desglosar dependiendo del lugar del frente en el que desarrollaron su inestimable labor:

- Inspección de campos de prisioneros en Alemania: Esta comisión estuvo formada por un número de 31 oficiales de distintas Armas (Infantería, Caballería e Ingenieros). Los médicos militares que participaron fueron Julio del Castillo y Domper, Servando Barbero Saldaña, José Barreiro de la Iglesia, Antonio Ferratges Tarrida (Figura 2), José Romero Aguilar, Fernando Pérez García, Enrique Ostalé González y Antonio Vallejo Nágera.

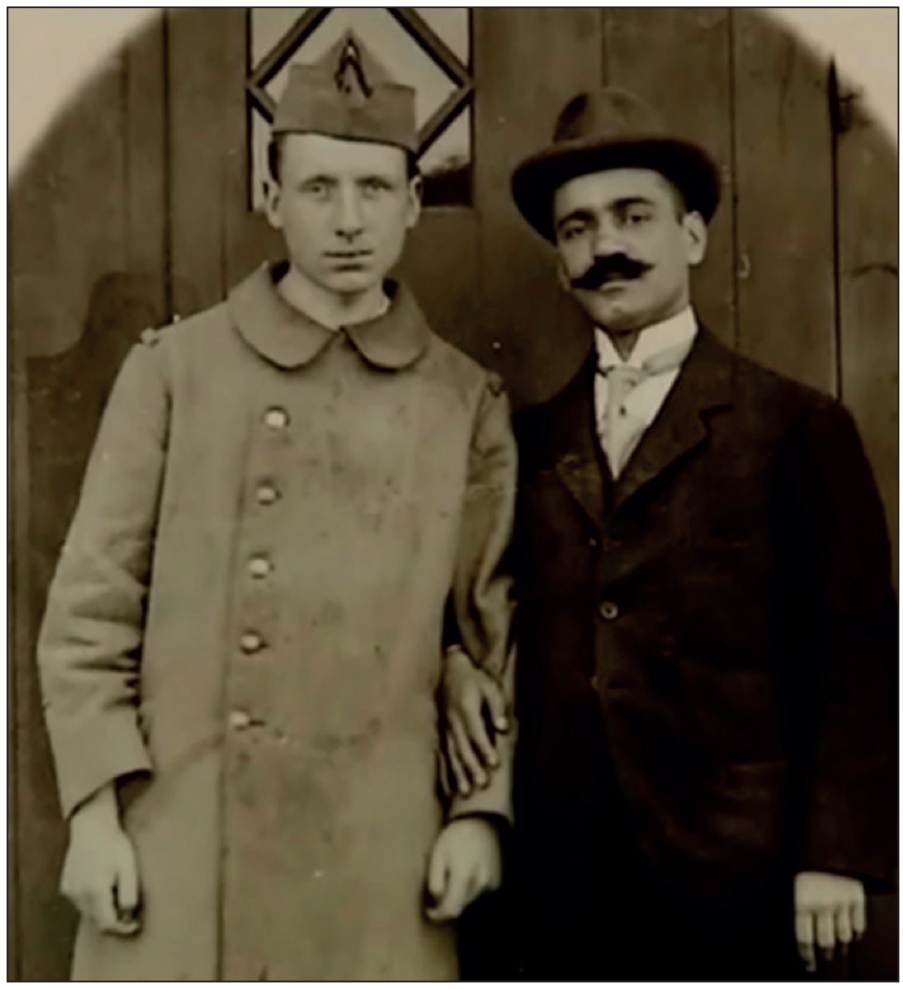

Figura 2. Un prisionero belga junto al capitán médico Antonio $\mathrm{Fe}$ rratges, comisionado a Alemania durante la primera guerra mundial. Imagen modificada por los autores tomada del documental de canal historia: "Alfonso XIII redentor de cautivos".

- Inspección de campos de prisioneros en Austria y Hungría: Esta comisión estuvo presidida entre marzo de 1917 y enero de 1918 por el comandante de Ingenieros Ricardo Álvarez-Espejo y Castejón. A ella pertenecieron entre otros, los capitanes médicos Alberto Blanco Rodríguez, Antonio Jiménez Arrieta y Fidel Pagés Miravé.

- Misión permanente en París: Francia y los frentes inglés y francés fueron posiblemente los más visitados por los observadores españoles. A esta comisión permanente pertenecieron un número variable de oficiales venidos desde España por un tiempo más o menos prolongado. Entre ellos se encontraba el farmacéutico $1^{\circ}$ Antonio Moyano Cordón y el capitán médico Mariano Gómez Ulla ${ }^{5}$.

- Inspección de campos de prisioneros en otros países: También se efectuaron visitas ocasionales a campos situados en Bulgaria y Turquía ${ }^{4}$.

A modo de ejemplo, describiremos la actuación de tres de estos médicos militares españoles que fueron desplegados en distintos puntos del frente europeo durante la primera guerra mundial (Figura 3):

\section{Capitán médico Mariano Gómez Ulla}

La experiencia europea de Mariano Gómez Ulla había comenzado unos años antes del inicio de la primera guerra mundial. En febrero de 1912 el general Orozco le concedió una comisión indemnizable de tres meses para París, Berlín y 
Roma, con el fin de estudiar las organizaciones del servicio de sanidad militar en las tres naciones. «Vio todo, estudió con detalle toda la organización sanitaria extranjera, discutiendo sus ventajas e inconvenientes, hablando de nuestros servicios y material, hasta el extremo de que los italianos desconocían nuestra artola sencilla y les hubo de mandar un diseño de ella. En las tres naciones dejó muy alto el nombre de la Sanidad Militar española, haciendo grandes amistades con compañeros de los servicios sanitarios extranjeros.»

En septiembre de 1915, se le destina en comisión a las órdenes del embajador de España en Berlín, para inspeccionar los campos de prisioneros en Alemania. Durante su estancia aprovecha para estudiar los servicios sanitarios desplegados en el conflicto. En junio de 1916, tras la petición del káiser al rey de España, es destinado a inspeccionar los campos de oficiales alemanes prisioneros en Francia. Una vez terminado este cometido, queda agregado a una comisión de nuestro estado mayor para estudiar la campaña en los frentes de Francia y Bélgica.

En agosto de 1917 asciende al empleo de comandante médico, siguiendo en la comisión y continúa con el estudio de los servicios sanitarios de ambos bandos beligerantes. Se dedica especialmente a analizar las organizaciones quirúrgicas y sobre todo, las evacuaciones desde la primera línea hasta la retaguardia a través de los diferentes escalones sanitarios. Compara las ventajas e inconvenientes de los diferentes modelos. «Salvo en el alemán, en que el espíritu organizador alcanzó a todos los estratos de sus elementos, todos los servicios sanitarios de los ejércitos beligerantes fracasaron de la manera más rotunda y lamentable en sus actuaciones, siendo necesario rehacerlos de nuevo y sobre la marcha, creando organizaciones y trazados de servicios y sobre todo, aportando un caudal de elementos de curación, de instrumental, transporte y personal adiestrado en proporciones gigantescas por lo insospechado»
En el teatro de operaciones francés visita los campamentos de prisioneros y es allí, donde formando parte de las ambulancias quirúrgicas ligeras, observa y actúa al lado de grandes maestros de la cirugía francesa ${ }^{6}$.

\section{Capitán médico Fidel Pagés Miravé}

Otro médico militar español que también fue desplegado durante la primera guerra mundial fue Fidel Pagés Miravé. Este médico ha pasado a la historia de la medicina mundial por ser el primero en describir la técnica de la anestesia epidural en 1921. En febrero de 1916, según R.O. del día 24, se le concede una comisión de servicio para trasladarse a Austria-Hungría, por tiempo indefinido. Salió desde Madrid el día 11 de abril de ese año. Su prestigio como cirujano y sus conocimientos de francés y alemán, le permitieron realizar este cometido. Su misión en el imperio austro-húngaro sería la de cooperar como delegado del embajador de España en Viena, en la inspección de los campos de prisioneros de guerra internados en aquella nación. Durante su comisión realizó inspecciones en todos los campos situados en Hungría en colaboración con el profesor Werner, hasta que en agosto de 1916 pasó a la ciudad de Viena adscrito como cirujano a la sección de prisioneros del Hospital Militar $n^{\circ} 2$ de esta ciudad. En ese centro llevó a cabo diferentes operaciones quirúrgicas, según consta por los certificados expedidos por el jefe de la clínica quirúrgica, visados por el director del hospital. También formó parte en misiones de traslados de heridos de guerra valorando los medios de evacuación de la época. La falta de alimentos, cuidados elementales e higiene posiblemente fueron los causantes de que enfermase gravemente, teniendo que ser repatriado a España ${ }^{7-9}$.

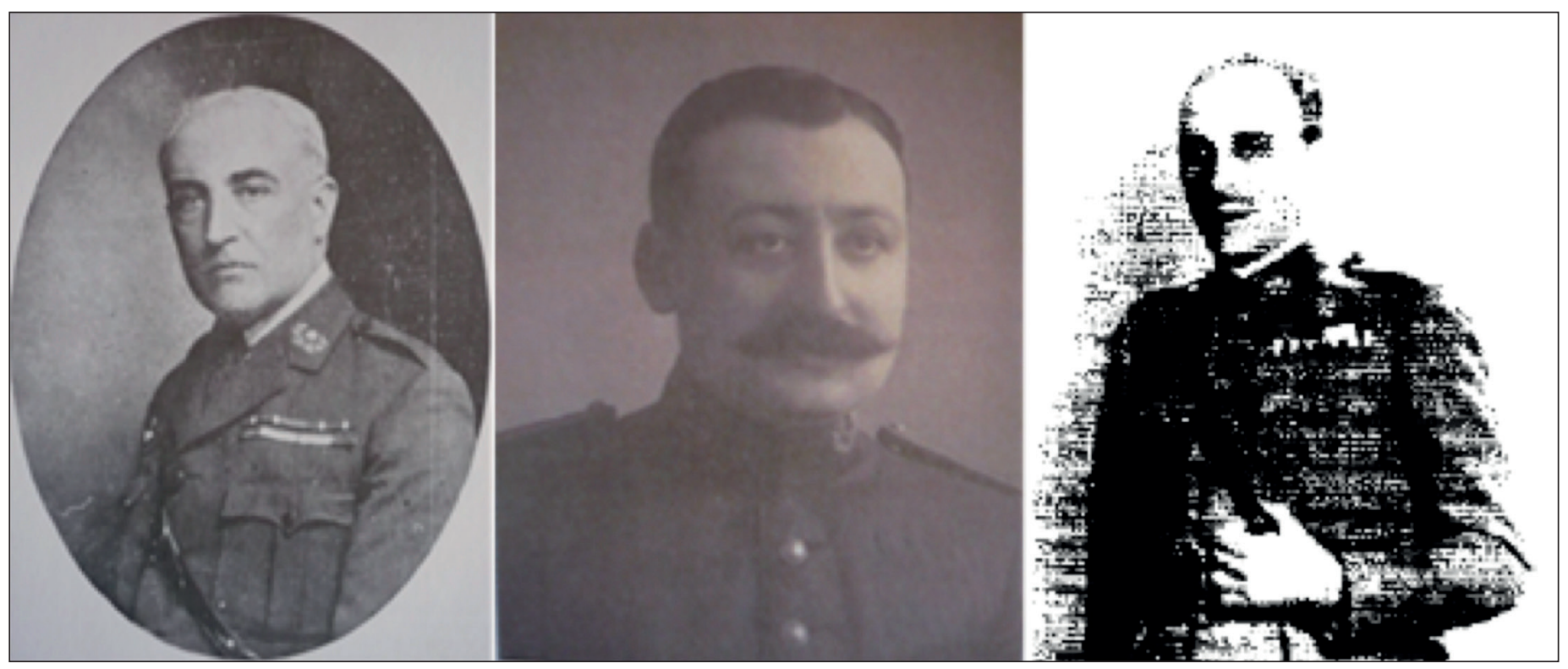

Figura 3. Tres de los oficiales médicos españoles comisionados al frente europeo durante la primera guerra mundial. D. Mariano Gómez Ulla (Gómez Ulla M. Mariano Gómez Ulla: un hombre, un cirujano, un militar. Madrid: 1981, p 2), D. Fidel Pagés Miravé (imagen tomada de Fidel Pagés Miravé y otros sanitarios militares ejemplares. Ministerio de Defensa; 2008. p 17) y D. Antonio Vallejo Nágera (imagen tomada de Abejaro de Castro LF. Historia de la psiquiatría militar española 1800-1970. [Tesis doctoral]. Madrid: Universidad Complutense; 1993). 


\section{Capitán médico Antonio Vallejo Nájera}

Este eminente psiquiatra fue designado para formar parte de la comisión militar agregada a la embajada de España en Berlín debido fundamentalmente a su conocimiento del idioma alemán.

Durante 1917-1919, este capitán médico pudo observar en los centros asistenciales de Alemania y Austria, los adelantos psiquiátricos tanto científicos como asistenciales de la época. Aprovechó su estancia en estos países para visitar clínicas psiquiátricas, destacando las pertenecientes a las universidades de Munich, Heidelberg y Viena. En ellas tuvo la oportunidad de asistir a las lecciones de E. Kraepelin, W. Gruhle (cuya obra tradujo posteriormente al castellano) y W. von Jauregg (poco después premio Nóbel).

Su personalidad científica adquirió a partir de este momento una formación psiquiátrica profundamente germánica. Asimiló su mentalidad nosológica, etiopatogénica, terapéutica y en suma médica y organicista de la especialidad, que marcaría en el futuro toda su obra escrita, producción investigadora y actividad docente ${ }^{10}$.

\section{MÉDICOS MILITARES ESPAÑOLES EN GUINEA ECUATORIAL}

El territorio continental de Guinea limita al norte con Camerún, que por entonces pertenecía al imperio alemán. Durante el conflicto de la primera guerra mundial los combates se extendieron a las colonias en África y Asia. Las tropas alemanas perdieron frente al ejército francés su dominio sobre el territorio camerunés y se vieron forzadas a atravesar la frontera sur, internándose en Guinea, que en aquella época pertenecía al reino de España.

La situación era crítica pues fueron más de 17.000 personas (civiles y militares alemanes junto con las tropas africanas y sus familias) las que se refugiaron en la colonia española (Figura 4). Las autoridades ordenaron el envío de fuerzas de Infantería de

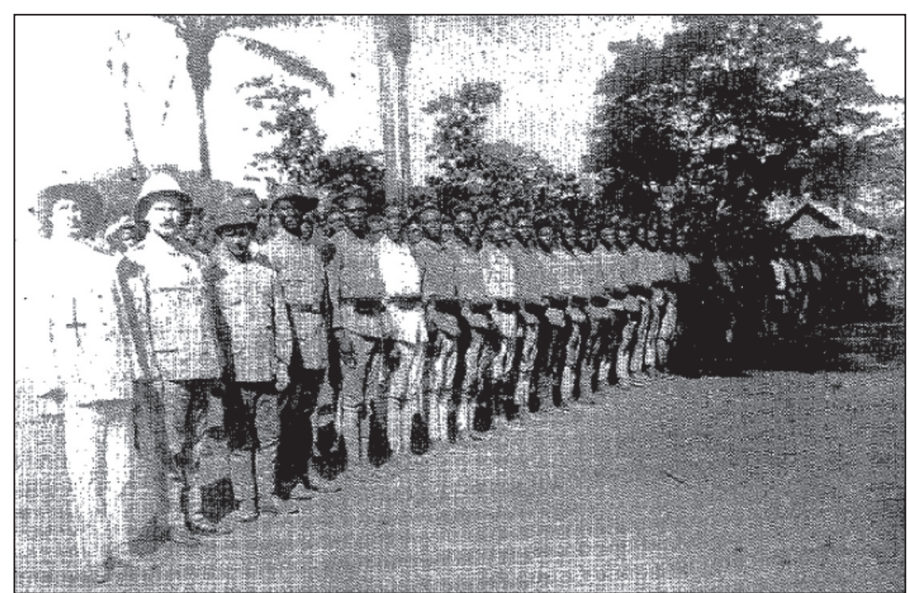

Figura 4. Tropas alemanas (occidentales e indígenas) procedentes de Camerún en Guinea. Imagen tomada de Martínez M. El servicio sanitario en los campamentos de internamiento de alemanes en Fernando Poo durante la primera guerra mundial, meritoria intervención de la sanidad naval (1916-1918). Revista General de Marina 1995; (228): 176-97.
Marina ${ }^{11}$, junto con tres oficiales médicos y siete practicantes de sanidad de la Armada que colaboraron con los tres médicos ya destinados en la colonia (Figura 5). Los oficiales médicos eran Antonio González Romero, Rodrigo Suárez Zamora, Horacio Olivares Bell, Luis Figueras Ballester, Luis Mena Burgos y Plácido Huertas Naves ${ }^{12}$ (Figura 6). Estos médicos contaron con la colaboración del Dr. Hilfrich (médico alemán) y con los practicantes alemanes e indígenas. Por motivos de seguridad y sanitarios, se decidió traslado de los refugiados hasta la isla de Fernando Poo. La población europea pronto fue evacuada hacia España, sin embargo la mayoría de los africanos permaneció en la colonia española.

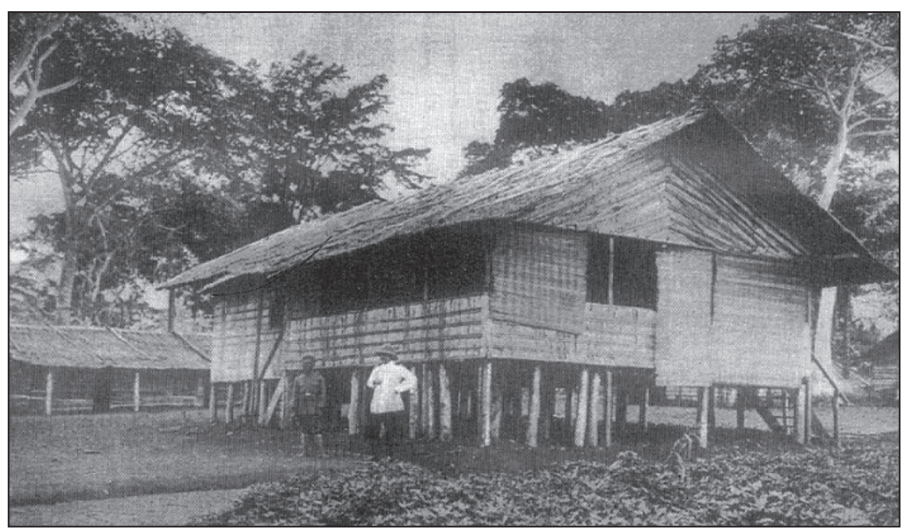

Figura 5. Casa del médico militar destinado en Guinea. Imagen tomada de Martínez M. El servicio sanitario en los campamentos de internamiento de alemanes en Fernando Poo durante la primera guerra mundial, meritoria intervención de la sanidad naval (19161918). Revista General de Marina 1995; (228): 176-97.

El campamento de los refugiados se fue edificando progresivamente. Se construyeron enfermerías dotadas con una sala general, sala de operaciones, farmacia, laboratorio, despensa y cocina. También se disponía de una policlínica y de una enfermería de infecciosos. Se realizan importantes medidas de salud pública como son: dotar el campo de agua potable, construir letrinas que son sometidas constantemente a la acción del fuego para desinfectar la excreta y que aprovecha el humo para impedir que los insectos sirvan como vectores de infecciones, trazar desagües que limitan el encharcamiento de las aguas, desbrozar el boque, sembrar huertas que permiten que el indígena aumente su dieta en contenido y variabilidad. Destacamos las medidas de prevención de la malaria utilizada en este campo: la quinina tomada a dosis preventiva por el personal europeo a dosis de 1 gramo cada 4 días, el constante uso de mosquiteros y el uso de agua filtrada junto con una buena alimentación. Por ultimo mencionar que se llevó a cabo una campaña de vacunación en masa de todos los internados con dosis de vacuna antivariólica facilitada por el Instituto de Higiene «Alfonso XIII» ${ }^{13}$.

A pesar de los riesgos originados por el hacinamiento de los refugiados, la morbilidad no superó los 100 enfermos/día, siendo los procesos más frecuentes en un primer momento las afecciones respiratorias y la gripe, y posteriormente los procesos gastrointestinales. Entre los europeos hubo 3 casos de fallecimiento, mientras que entre los africanos la cifra alcanzó un 320 por mil el primer mes, descendiendo luego a 25 por 1000 y posteriormen- 

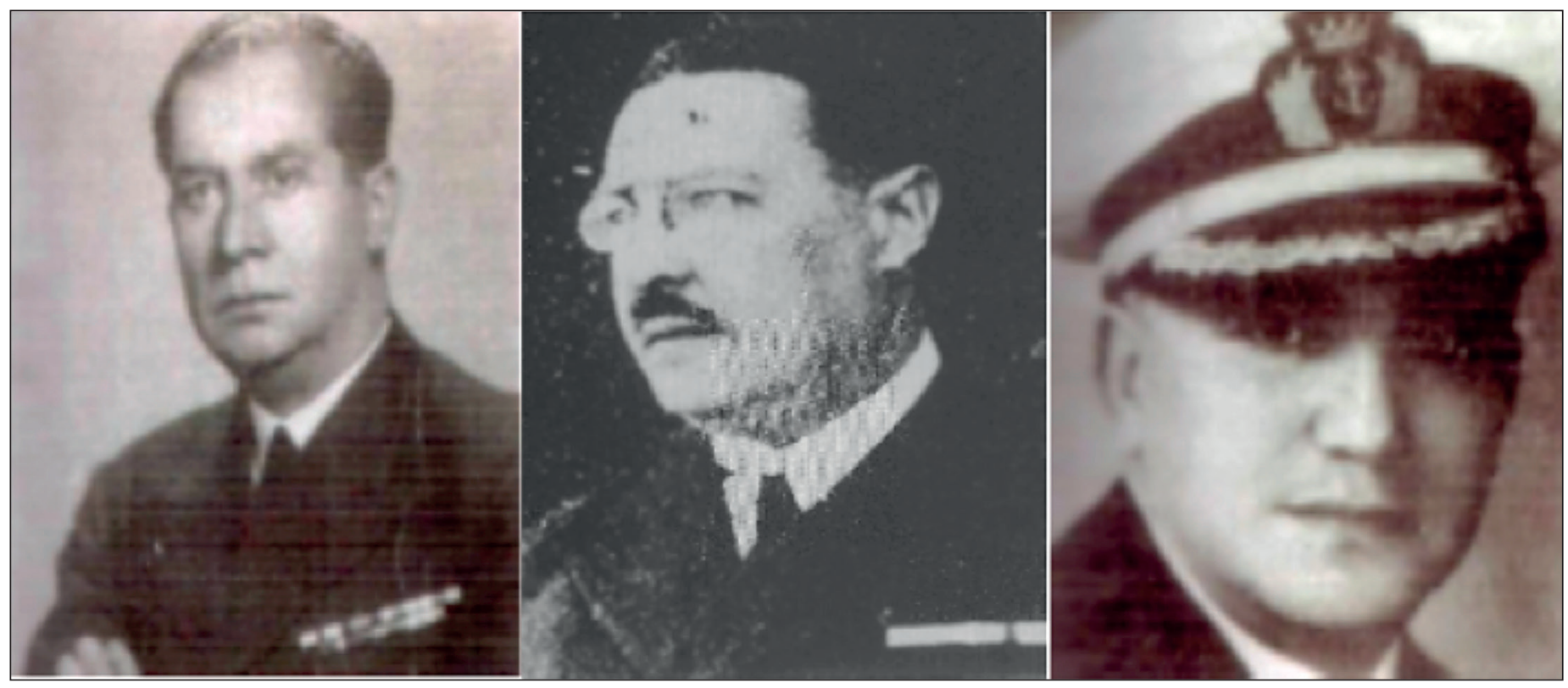

Figura 6. Tres de los médicos españoles pertenecientes al cuerpo de sanidad de la armada. D. Rodrigo Suárez Zamora, D. Luis Figueras Ballester y D. Luis Mena Burgos. Imagen tomada de Martínez M. El servicio sanitario en los campamentos de internamiento de alemanes en Fernando Poo durante la primera guerra mundial, meritoria intervención de la sanidad naval (1916-1918). Revista General de Marina 1995; (228): 176-97.

te se estabilizó en 7 por mil; cifras que los médicos de la época consideraban mejores que las que habitualmente se presentaban en el continente africano. Entre las causas de esta mortalidad destaca la disentería, las infecciones broncopulmonares y la inanición. Gracias a las memorias del Dr. Figueras podemos conocer las principales enfermedades observadas en los refugiados: viruela, gripe, tuberculosis, lepra, sarna, caries, enfermedades venéreas, paludismo y disentería. En total se estima que el personal médico atendió a cerca de 12.000 refugiados ${ }^{13,14}$.

Mientras que las tropas de Infantería de Marina que habían reforzado el dispositivo de seguridad fueron repatriadas en marzo de 1917, los oficiales médicos permanecieron en la colonia desarrollando su misión hasta el término del conflicto. Incluso un coronel médico, D. Estanislao Lluesma llegó a ser gobernador general de Guinea entre 1934 y $1935^{12}$.

\section{DISCUSIÓN}

Desde el punto de vista de sanidad militar, la primera guerra mundial supuso un punto de inflexión. La globalización del conflicto, la aparición de nuevos tipos de armamento que ocasionaron un gran número de bajas en combate, el empleo de armas químicas por ambos bandos, las condiciones de vida de los militares en una guerra predominantemente de trincheras y un despliegue sanitario frecuentemente ineficaz son algunos de los factores que motivaron el cambio de la doctrina sanitaria. De los 60 millones de efectivos movilizados por los países en conflicto, 7 millones murieron y aproximadamente 19 millones fueron heridos (medio millón amputados) en combate ${ }^{15}$.

La Artillería ocupa un papel predominante en los conflictos desde la primera guerra mundial. Este hecho provocó que aumentaran las bajas con heridas de metralla frente a las que presentaban lesiones por arma de fuego ${ }^{16}$. El Dr. Cooper y su equipo ${ }^{17}$ contabilizaron que el $61 \%$ de las bajas fueron por artillería respecto al $39 \%$ por arma de fuego. En el estudio de Barnett ${ }^{18}$ se detalla el agente lesional que ocasionó las bajas desglosadas por algunos de los ejércitos que participaron en este conflicto. (Tabla 1). Su estadística describe importantes diferencias entre bandos. Esto puede ser debido a una recogida incompleta de los datos (los obtenidos del ejército alemán están basados en un análisis de 14.486 bajas, que suponen menos del 1\% de todas las bajas alemanas durante el conflicto) y al empleo de distintas tácticas (los alemanes pudieron emplear artillería tanto en operaciones de defensa como de ataque, mientras que las tropas estadounidenses emplearon en mayor medida armas propias de infantería ${ }^{16}$. Al igual que en conflictos posteriores, la región más afectada por lesiones penetrantes fue los miembros $^{19}(70 \%)$, seguida de la cabeza y el cuello $(17 \%)$, el tórax

Tabla 1. Heridos y muertos clasificados por ejército de pertenencia y agente lesional.

\begin{tabular}{|c|c|c|c|c|c|c|}
\hline & \multicolumn{2}{|c|}{ Alemanes 1914-17 } & \multirow{2}{*}{$\begin{array}{c}\text { Británicos } \\
\text { Heridos }\end{array}$} & \multicolumn{2}{|c|}{ Estadounidenses 1918} & \multirow{2}{*}{$\begin{array}{c}\text { Franceses } 1918 \\
\text { Heridos }\end{array}$} \\
\hline & Heridos & Muertos & & Heridos & Muertos & \\
\hline Balas & $51 \%$ & $39 \%$ & $39 \%$ & $14 \%$ & $8 \%$ & $30 \%$ \\
\hline Fragmentos* & $46 \%$ & $56 \%$ & $61 \%$ & $85 \%$ & $92 \%$ & $58 \%$ \\
\hline
\end{tabular}

*Incluye metralla, granadas y bombas. Se desconocen los datos de fallecidos británicos y franceses en esta serie. Tomado de: Barnett G. Caring for the casualties. WWII History 2008; 4:40-7. 
(4\%), abdomen (2\%), el 7\% restante incluye heridas en glúteos y dorso $^{20}$. Es en esta contienda donde aparecen por primera vez las heridas anfractuosas y los grandes destrozos que produce la metralla de artillería y de aviación, lesiones nada comparables a las producidas por una bala de fusil ${ }^{5}$.

Se puede afirmar que la guerra química comenzó durante esta contienda. Ambos bandos emplearon más de 30 sustancias químicas como armas. Se calcula que estos agentes causaron aproximadamente 1.300 .000 bajas durante el conflicto, de las cuales 91.000 fueron mortales. Una de las lecciones identificadas es que la eficacia de las armas químicas no se puede medir únicamente por el número de bajas que ocasiona, sino por el importante efecto psicológico sobre el combatiente ${ }^{21}$.

La primera guerra mundial está considerada como una contienda de trincheras. Esta situación provocó con frecuencia hacinamiento y desastrosas medidas higiénicas entre los combatientes de uno y otro bando. A modo de ejemplo, Alsina ${ }^{22}$ describe el apoyo sanitario durante la batalla de Verdún de 1916. En su artículo destaca la presencia incontrolable de ratas que transmitieron hantavirosis, la infestación por piojos que fueron el principal vector de la rickettsia y la aparición del «pie de trinchera» favorecida por la exposición prolongada a la humedad, al frío, junto con las malas condiciones higiénicas y el mal estado del vestuario y calzado de los militares allí desplegados.

Desde un punto de vista logístico sanitario, este conflicto modificó en gran medida el despliegue sanitario y los medios de evacuación. Campillo ${ }^{23,24}$ analiza esta circunstancia destacando que las unidades sanitarias tuvieron que desplegar más retaguardia para evitar el alcance de la artillería enemiga, se demostró que el resultado del tratamiento era mejor cuanto menor era el tiempo transcurrido entre la producción de la lesión y el tratamiento quirúrgico y se constató la necesidad de contar con un servicio de sanidad único, indivisible y coordinado con la misión de recoger al herido del campo de batalla, prestarle los cuidados más urgentes, prepararlo para el transporte a un lugar fuera del alcance del fuego enemigo y conseguir que llegase en las mejores condiciones a la primera formación de tratamiento, donde se le pudiera realizar la intervención quirúrgica pertinente o someterle a una nueva evacuación hacia un escalón sanitario superior. Además, aparece la necesidad de regular la acumulación de bajas en retaguardia, siendo necesaria una rigurosa clasificación de los heridos y enfermos en cada escalón.

La Medicina Militar consiguió grandes avances durante la primera guerra mundial. Se despliegan laboratorios móviles de campaña, los aparatos de radiografía se hacen más ligeros y portátiles, se emplea suero salino intravenoso de forma protocolizada, en quirófano se comienza a utilizar pinzas hemostáticas, jeringas subdérmicas, termómetros y luz eléctrica en las lámparas quirúrgicas. Se irrigan suero salino y antisépticos directamente sobre la herida, se procede a desbridamientos amplios en las lesiones. Se diseña la férula de tracción de Thomas para fracturas femorales, disminuyendo notablemente la tasa de amputación precoz. Comienza el diseño de prótesis y la creación de centros de rehabilitación especializado en el tratamiento del militar herido. El amplio número de lesiones en cara provoca el desarrollo de la cirugía maxilofacial y plástica ${ }^{15,25-28}$. La transfusión de sangre completa gracias al descubrimiento del citrato como anticoagulante se empleó de forma habitual en la primera guerra mundial, siendo considerado por algunos autores ${ }^{25,26}$ como el mayor avance médico de este conflicto ${ }^{29,30}$. La medicina preventiva también tuvo una importancia sobresaliente. Se introducen la vacuna antitetánica, antitifoidea y se mejoró el tratamiento frente a la disentería y malaria. Sin embargo, la epidemia de gripe fue devastadora en ambos bandos ${ }^{15}$.

El tema de los observadores militares españoles en la primera guerra mundial ha recibido poca atención por parte de los investigadores. Se realizaron 2.538 visitas a 1.456 instalaciones distintas tales como hospitales y campos de prisioneros ${ }^{4}$. Sus apreciaciones provocaron un importante efecto sobre la Doctrina del Ejército y la Armada Española. Muchos de estos oficiales participaron especialmente durante el período 1924-1930 en la redacción de los reglamentos que renovaron los textos oficiales de instrucción y campaña 5 . Por otra parte, la personalidad de gran parte de estos oficiales (por su prestigio profesional o por su papel en la historia de España) ofrece un gran interés. Este es el caso de los oficiales médicos desplegados en los diferentes frentes, cuyas lecciones logísticas y asistenciales aprendidas en la primera guerra mundial, se pusieron más tarde en práctica durante los conflictos en el norte de África ${ }^{31,32}$, en la guerra civil española ${ }^{33}$ o durante la segunda guerra mundial ${ }^{34}$ y sirvieron como doctrina para los alféreces alumnos médicos destinados en la Academia de Sanidad 6 . Se diseña un Puesto Quirúrgico Avanzado Móvil (PQA) y desplegable en el frente ${ }^{35}$, se comienzan a emplear aviones sanitarios para el transporte de heridos a los hospitales de retaguardia, se crean aeródromos cercanos a la línea de contacto con el enemigo ${ }^{36}$ e incluso se comienza a habilitar un buque como hospital (varios barcos alemanes internados en puertos españoles durante el conflicto, fueron transferidos a nuestra Armada como compensación por las pérdidas españolas sufridas tras los ataques de submarinos alemanes. El «Crefeld», uno de dichos buques alemanes fue transformado en hospital. Se comenzó a recibir cientos de colchonetas, unos cuadros de hamacas y aparatos de suspensión de Linsweyler. Se planeó dotarlo de 253 camas, junto con un reglamento de organización interior e incluso se liberó una cantidad de 1.200 .000 pesetas (a pesar del esfuerzo realizado este proyecto finalmente no se llevó a cabo) ${ }^{12}$.

La oficina de procautivos creada por el rey Alfonso XIII en el Palacio Real de Madrid destinada a una labor humanitaria merece un reconocimiento especial. Apenas 54 funcionarios atendieron 157.851 casos (141.291 de información a familias y 16.560 de repatriación de militares) y se obtuvieron 102 indultos ${ }^{3}$.

Desgraciadamente durante la realización de la misión encomendada en este conflicto, dos oficiales médicos españoles fallecieron en acto de servicio presumiblemente a consecuencia de las enfermedades contraídas en los campos de prisioneros inspeccionados. Concretamente fueron el teniente coronel médico Julio del Castillo y Domper y el capitán médico Fernando Pérez García ${ }^{4}$.

Por esta brillante actuación durante la primera guerra mundial, muchos de los oficiales médicos españoles fueron condecorados por el ministerio de guerra, por el comité internacional de la cruz roja y por varios gobiernos extranjeros. Como ejemplo, a Mariano Gómez Ulla se le concedió la medalla del reconocimiento francés, la Orden de la Estrella Negra y la Cruz Militar de primera clase de Bélgica ${ }^{6}$. A Fidel Pagés Miravé, se le autoriza para usar sobre el uniforme la Medalla de Segunda Clase de cruz roja española debido a su comportamiento en los campos de pri- 


\section{Una «hazaña prácticamente desconocida»: la participación de médicos militares españoles en la ...}

sioneros del imperio austro-húngaro ${ }^{8}$. Por su parte, a Antonio Vallejo Nágera, se le concedió la Cruz de Primera Clase al Mérito Militar con Distintivo Blanco, la Cruz de Isabel La Católica $\mathrm{y}$ varias condecoraciones de los gobiernos francés y belga ${ }^{10}$. Así mismo, para recompensar la excepcional labor de los médicos de la Armada durante su acción humanitaria en un ambiente sanitario clásicamente tan complicado como era Guinea Ecuatorial $^{37}$, se les concedió la Cruz Blanca del Mérito Naval ${ }^{14}$.

\section{CONCLUSIÓN}

Hablar hoy en día en España de misión humanitaria no nos sorprende, sin embargo a principios del siglo XX era una práctica inusual. Podemos considerar que la participación durante la primera guerra mundial de médicos militares españoles en el frente europeo y la asistencia de refugiados en Guinea Ecuatorial, es una heroicidad que se debe divulgar y admirar. Las experiencias sanitarias y logísticas aprendidas durante el conflicto supusieron una experiencia de enorme importancia que tuvo gran repercusión en nuestra sanidad militar. El artículo pretende valorar el trabajo desempeñado por aquellos oficiales médicos. Una «hazaña prácticamente desconocida» que no debe ser olvidada.

\section{BIBLIOGRAFÍA}

1. Lozano A. Breve historia de la primera guerra mundial. Madrid: Ediciones Nowtilus; 2011.

2. Gilbert M. La primera guerra mundial. Barcelona: Historia del siglo XX Ediciones; 2004.

3. Pando J. Un rey para la esperanza, la España humanitaria de Alfonso XIII en la gran guerra. Madrid: Ediciones Temas de hoy; 2002.

4. Marín FA. Martínez se va a la guerra, intervenciones militares españolas en el extranjero. Barcelona: Inédita Editores; 2005.

5. Redondo F. Los observadores militares españoles en la Primera Guerra Mundial. Revista de historia militar 1985; 59:197-208

6. Gómez Ulla M. Mariano Gómez Ulla: un hombre, un cirujano, un militar. Madrid: 1981

7. Velázquez Rivera I. Aproximación a la obra científica del Comandante médico Fidel Pagés Miravé. Sanid mil 2011; 67(S): 193-238.

8. Velázquez Rivera I, Diz Rodríguez R, Robledo Aguilar A. Fidel Pagés Miravé, cirujano militar. Med mil 2003; 59(3): 51-5.

9. Belaústegui A. Fidel Pagés Miravé y otros sanitarios militares ejemplares. Ministerio de Defensa; 2008.

10. Abejaro de Castro LF. Historia de la psiquiatría militar española 1800-1970. [Tesis doctoral]. Madrid: Universidad Complutense; 1993.

11. Cervera J. Presencia y esfuerzo: la Infantería de Marina en Guinea Ecuatorial. Revista General de Marina 2012; 11-30.
12. Gracia Rivas M. La Sanidad Naval española, Barcelona: Bazan; 1995.

13. Plaza JF, Arcéiz JL. La Sanidad de la Armada durante la internación alemana en Fernando Poo en la Primera Guerra Mundial. En: Bicentenario del Hospital de San Carlos. Madrid: Ministerio de Defensa; 2009. p. $125-51$.

14. Martínez M. El servicio sanitario en los campamentos de internamiento de alemanes en Fernando Poo durante la primera guerra mundial, meritoria intervención de la sanidad naval (1916-1918). Revista General de Marina 1995; (228): 176-97.

15. Gabriel RA, Metz KS. A history of military medicine, Connecticut: Greenwood Press; 1992

16. Navarro Suay R, Hernández-Abadía de Barbará A, Gutiérrez Ortega C, Tamburri Bariain R, Bartolomé Cela E, Gilsanz Rodríguez F. Análisis del agente lesivo en la baja de combate. Sanid mil 2011; 67(1):18-24.

17. Cooper G, Gotts P. Ballistic protection. En Mahoney P, Ryan J, Brooks A, Schwab C, editors. Ballistic trauma. $2^{\circ}$ ed. Springer; 2004. 67-89.

18. Barnett G. Caring for the casualties. WWII History 2008; 4:40-7.

19. Navarro Suay R, Hernández-Abadía de Barbará A, Gutiérrez Ortega C, Bartolomé Cela E, Tamburri Bariain R, Gilsanz Rodríguez F. Análisis de la topografía lesional en la baja de combate. Sanid Mil 2011; 67(2): 78-83.

20. Cutting P. Surgery in the camp. En Mahoney P, Ryan J, Brooks A, Schwab C, editors. Ballistic trauma. $2^{\circ}$ ed. Springer; 2004. 585-91.

21. Pita R. Armas químicas, la ciencia en manos del mal, Madrid: Plaza-Valdés; 2008.

22. Alsina Álvarez J. Verdún, un siglo después. Med Mil 2005; 61(1): 43-9.

23. Campillo J.R. Bases históricas del escalonamiento del Servicio de Sanidad en operaciones. Sanid. Mil. 2008; 64 (1):43-51.

24. Campillo Laguna JR. Norma de Evacuación, clave en el despliegue sanitario. Med Mil 2004; 60(3): 185-190.

25. Wright J. A history of war surgery, Gloucestershire: Amberley Publishing; 2011.

26. Scotland TR, Heys SD. War surgery 1914-18, Berkshire: Helion\&Company; 2012.

27. Pöll JS. The anaesthestist 1890-1960, a historical comparative study between Britain and Germany, Breda: Erasmus Publishing; 2011.

28. Condon-Rall. A brief history of military anesthesia. En: Zajtchuk R, Bellamy R, editors. Anesthesia and perioperative care of the combat casualty. Washington: Textbook of Military Medicine; 1995.p. 855-96.

29. Hess JR, Thomas MJG. Blood use in war and disaster: lessons from the past century. Transfusion 2003; 11(43): 1622-33.

30. Starr D. Historia de la sangre, Barcelona: Sine Qua Non; 2000.

31. Martínez FJ. Entre la diplomacia médica y la política sanitaria: médicos militares en el protectorado español en Marruecos (1906-1927). Revista de historia militar 2012; 203-42.

32. Navarro R, Plaza JF. 1925: Cuando volvimos a ser grandes...el apoyo sanitario en el desembarco de Alhucemas. Sanid mil 2012; 68(4): 247-56.

33. Masson P. Historia de Sanidad Militar española, Barcelona: Ediciones Pomares-Corredor; 1994.

34. Bescos J. Misiones de la Sanidad Militar española en el extranjero: la Sanidad Militar en la División Azul. Med Mil.1995; 51(2): 184-93.

35. Martín Sierra F. Hospital quirúrgico de montaña «Gómez Ulla». Medicina Militar 2000 (56) Num 2, 117-21.

36. Diz Rodríguez MR, Vírseda Chamorro M, Diz Rodríguez L, Cancho Gil MJ, Sáez de Benito D, Paños Lozano P. El transporte de heridos vía aérea durante la campaña del norte de África 1921-1927. Med mil 2006; 62(1): 41-7.

37. De Granda Orive J. Sanidad Militar y aspectos sanitarios en Fernando Poo durante el gobierno de los brigadieres (1859-1869). Sanid mil 2011; 67(1):53-62. 